\title{
Abdominal Retractor and Intraoperative Arrhythmia
}

\author{
Arpita Das ${ }^{1}$, Sarika Sudhir Naik², Dhananjay Reddy ${ }^{3}$
}

\begin{abstract}
Intraoperative arrhythmias are frequently seen. Mostly during cardiac surgeries and general anesthesia. Ventricular premature complexes (VPCs) constitute 15\% of these arrhythmias and are benign in nature. However, if VPCs are persistent and not treated on time, can lead to life-threatening arrhythmia. We discussed a case report of 37-year-old woman with no preexisting comorbidities, when administered regional anesthesia for abdominal hysterectomy, had repeated VPCs. Arrhythmias were related to abdominal retractor position and were aborted when the retractor was removed from the surgical area.

Keywords: Arrhythmia, Regional anesthesia, Retractor.

Research and Innovation in Anesthesia (2021): 10.5005/jp-journals-10049-0096
\end{abstract}

\section{BACKGROUND}

Arrhythmias are seen very frequently intraoperatively. Its occurrence has been reported in $70 \%$ of patients undergoing general anesthesia, ${ }^{1}$ mostly during cardiac surgeries, ${ }^{2}$ they are also seen in patients under central neuraxial blockade. ${ }^{3}$ Arrhythmias require attention and prompt treatment especially when it causes hemodynamic instability.

Ventricular premature complexes (VPCs) constitute 15\% of intraoperative arrhythmia, frequently observed during anesthesia with limited clinical significance. However, if VPCs are persistent and not treated on time, can lead to life-threatening arrhythmias. ${ }^{4}$

\section{Case Description}

A 37-year-old woman weighing $86.4 \mathrm{~kg}$ and body mass index (BMI) of 34.8 was scheduled to undergo a total abdominal hysterectomy for a large uterine fibroid. She was an American Society of Anaesthesiologists (ASA) 1 patient with no significant medical illness and previous surgical history. Airway assessment revealed a non-difficult airway. General examination was unremarkable with a heart rate (HR) between $80 \mathrm{bpm}$ and $90 \mathrm{bpm}$, regular rhythm, normovolemic, normotensive, and no pallor or pedal edema. All systemic examinations were within the normal limit. Intervertebral space palpation was difficult due to central obesity.

Routine blood investigation, 12-lead electrocardiograph (ECG), chest $\mathrm{X}$-rays, coagulation, and thyroid profile were all within normal limits. The patient was accepted for surgery and one unit packed cell was advised to keep in reserve for postoperative transfusion. Anesthesia planned was combined spinal and epidural anesthesia.

After taking informed consent, the patient was kept nil per oral for 6 hours. On the day of surgery, the patient was premedicated with inj. rabeprazole $20 \mathrm{mg}$ IV and inj. ondansetron $4 \mathrm{mg} 30$ minutes before surgery. Antibiotics inj. ceftriaxone, inj. amikacin, and inj. metronidazole were given 1 hour before surgery. After shifting the patient to the operation theater, all standard monitors were connected. Baseline vitals were noted. Baseline HR was 86 bpm, regular rhythm, NIBP was $118 / 74 \mathrm{~mm} \mathrm{Hg}$, oxygen saturation $\left(\mathrm{SpO}_{2}\right)-96 \%$ on room air, temperature $-36.6^{\circ} \mathrm{C} .18 \mathrm{G}$ intravenous cannula was inserted and ringer lactate started.

Regional anesthesia was given in the sitting position. Under all aseptic precautions and local infiltration with $2 \%$ plain lignocaine,

\footnotetext{
${ }^{1,2}$ Department of Anesthesia and Critical Care, Sri Sai Hospital, Bengaluru, Karnataka, India

${ }^{3}$ Department of Surgery, Sri Sai Hospital, Bengaluru, Karnataka, India

Corresponding Author: Arpita Das, Department of Anesthesia and Critical Care, Sri Sai Hospital, Bengaluru, Karnataka, India, Phone: +91 8193015979, e-mail: arpitadas2017new@gmail.com

How to cite this article: Das A, Naik SS, Reddy D. Abdominal Retractor and Intraoperative Arrhythmia. Res Inno in Anesth 2021;6(1):25-26.

Source of support: Nil

Conflict of interest: None
}

between L1 and L2 intervertebral space in Miesian approach using $16 \mathrm{G}$ Tuohy needle, the epidural catheter is inserted after confirming epidural space using loss of resistance (LOR) to saline and hanging drop method. To rule out intravenous and intrathecal positioning of epidural catheter, a test dose of $3 \mathrm{~mL}$ of $2 \%$ lignocaine and adrenaline 1:200,000 was given. Then, the subarachnoid block was given using $26 \mathrm{G}$ Quincke needle between $\mathrm{L} 3$ and $\mathrm{L} 4$ space in median approach and $3.0 \mathrm{~mL}$ of $0.5 \%$ heavy bupivacaine was given after confirmation of space by cerebrospinal fluid (CSF) outflow.

After the regional anesthesia, the patient was put in the supine position. The level of sensory block was T6. The vitals after 10 minutes of anesthesia were $\mathrm{HR}$ of $84 \mathrm{bpm}$, mean arterial pressure (MAP) of $80 \mathrm{~mm} \mathrm{Hg}, \mathrm{SpO}_{2} 97 \%$. The patient was stable for 30 minutes. After 30 minutes, the patient suddenly started complaining of nausea and vomiting, HR increased to $185 \mathrm{bpm}$ irregular rhythm, $\mathrm{P}$ wave present followed by intermittent bizarre QRS complex (diagnosed to be VPCs). The patient was hemodynamically stable and all other vitals were within normal limits. Immediately inj. dexamethasone $8 \mathrm{mg}$ IV as an antiemetic, inj. lignocaine $1.5 \mathrm{mg} / \mathrm{kg}$ IV, and inj. metoprolol $5 \mathrm{mg}$ slow IV given. Heart rate decreased to 140 , arrhythmias were still persistent, and MAP $>65 \mathrm{~mm} \mathrm{Hg}$. The surgeon was informed and was requested to clear the surgical field of any traction. Tractional force withdrawn keeping retractor in place, forceps holding ligaments, and tubular structures released.

Position of epidural catheter checked by negative aspiration of blood or CSF, arterial blood gas analysis was done there was no acidosis, all electrolytes were normal except potassium (3.1 $\mathrm{mmol} / \mathrm{L})$, repeat serum potassium was done and it was normal,

(c) The Author(s). 2021 Open Access This article is distributed under the terms of the Creative Commons Attribution 4.0 International License (https:// creativecommons.org/licenses/by-nc/4.0/), which permits unrestricted use, distribution, and non-commercial reproduction in any medium, provided you give appropriate credit to the original author(s) and the source, provide a link to the Creative Commons license, and indicate if changes were made. The Creative Commons Public Domain Dedication waiver (http://creativecommons.org/publicdomain/zero/1.0/) applies to the data made available in this article, unless otherwise stated. 
inj. magnesium sulfate $1 \mathrm{~g}$ slow IV given. Surgery was kept on hold for 15 minutes, HR decreased and maintained between $110 \mathrm{bpm}$ and 135 bpm, persistent PVC between 20 times and 40 times/ minutes and BP maintained, hence surgery proceeded as planned. Throughout the surgery, the patient was hemodynamically stable without any pain. Once the closing of the surgical site started, the retractor was removed and $\mathrm{HR}$ immediately came down to $86 \mathrm{bpm}$, regular rhythm without any PVC, and post retractor removal there was no further arrhythmia throughout the remaining period of surgery. After the skin closure, the patient complained of pain and hence epidural top-up of $10 \mathrm{~mL}$ (inj. lignocaine $1 \% 5 \mathrm{~mL}$ and inj. bupivacaine $0.25 \% 5 \mathrm{~mL}$ ) was given.

The postoperative period patient was observed in ICU for the next 24 hours for any further arrhythmias. Epidural infusion of bupivacaine $0.125 \%$ with fentanyl $2 \mu \mathrm{g} / \mathrm{mL}$ started for postoperative pain management. Echocardiography was done and was normal. Postoperative motor power regained after 2 hours of completion of surgery and no further arrhythmias were noted.

\section{Discussion}

The cause of intraoperative arrhythmia can be divided into three groups: patient, anesthesia, and surgery-related factors. ${ }^{4}$ Patients with preexisting cardiac disease and intracranial disease especially subarachnoid hemorrhage have a higher incidence of arrhythmias. Geriatric patients undergoing thoracic surgery frequently have atrial fibrillation. This patient was a middle-aged female with no known comorbidities; hence, patient-related factors of arrhythmia are unlikely.

Incidence of arrhythmia is more during general anesthesia owing to tracheal intubation, anesthetic drugs, and sometimes a lighter plane of anesthesia. On the other hand, regional anesthesia is more to cause bradyarrhythmias due to pharmacological sympathectomy. Electrolyte imbalance and abnormal arterial blood gases like hypercarbia or hypoxemia are known to cause arrhythmia, but these factors are already ruled in our patient.

Surgical manipulation during cardiac surgeries can precipitate arrhythmias. Noncardiac surgeries, in most cases, lead to bradyarrhythmias due to traction on the peritoneum, ligament, and sometimes direct compression of the vagus nerve. Few cases have been reported where the patient had intraoperative arrhythmias during laparotomy. In one case, the patient had undergone laparotomy for the repair of diaphragm injury and had intraoperative ventricular bigeminy, and the cause of arrhythmia was found out to be the placement of a Deaver retractor in close proximity to the heart. ${ }^{5}$ In another case, the patient had atrial fibrillation during Whipple surgery and direct irritation of the diaphragm by retractor was the likely cause. ${ }^{6}$ In our patient, the position of the Deaver retractor as a cause of arrhythmia cannot be ruled out as the arrhythmia persisted with the retractor kept in situ, and with the removal of it, arrhythmia was aborted., ${ }^{7,8}$ Drugs like ondansetron and dexamethasone need special mention as cases of arrhythmia have been reported with them but in our case, these drugs were given either long before or after the commencement of arrhythmic episode.

New-onset VPC may progress to life-threatening ventricular arrhythmias, hence need prompt treatment. In our case, we encountered multiple VPCs intraoperatively which was managed with lignocaine and beta-blockers. Throughout the surgery, the patient was hemodynamically stable. In this case, unexplained multiple VPCs were encountered in an otherwise normal patient during surgery, and retraction by Deaver's retractor is the most likely cause. Few cases reported in the literature support this observation; however, lower abdominal surgery makes this a rare possibility.

Nevertheless, the experience of this case reaffirms that surgery can be continued in a hemodynamically stable patient with preparation for possible serious arrhythmias.

\section{References}

1. Forrest JB, Rehder K, Cahalan MK, et al. Multicenter study of general anesthesia. III. Predictors of severe perioperative adverse outcomes. Anesthesiology 1992;76(1):3-15. DOI: 10.1097/00000542-19920100000002.

2. Fisher DM. Preoperative cardiac dysrhythmias; diagnosis and management. Anesthesiology 1997;86(6):1397-1424. DOI: 10.1097/00000542-199706000-00026.

3. Sokolow M, Mcllroy B. Clinical radiology. 4th ed., New York: Lange; 1986. pp. 116-117.

4. Dua N, Kumra VP. Management of perioperative arrhythmias. Indian J Anaesth 2007;51:310-323.

5. Anand N, Anand M, Mathur AV, et al. Intraoperative ventricular bigeminy: can retractor be a cause. J Anaesthesiol Clin Pharmacol 2010;26(4):569-570.

6. Bindal M, Demir A. Intraoperative new-onset atrial fibrillation with a surprising reason in a whippe operation. GKDA Derg 2019;25(1):72-74.

7. Kasinath NS, Malak O, Tetzlaff J. Atrial fibrillation after ondansetron for prevention and treatment of postoperative nausea and vomiting: a case report. Can J Anaesth 2003;50(3):229-231. DOI: 10.1007/ BF03017789.

8. Rao G, Zikria EA, Miller WH, et al. Cardiac arrhythmia after dexamethsaone. JAMA 1972;222(9):1185. DOI: 10.1001/ jama.1972.03210090065040. 